\title{
La gestión pública de empresas: el desafío de un abordaje multinivel
}

\section{State-owned enterprises management: the challenge of a multilevel approach}

\author{
Agoff, Sergio Leandro; Blugerman, Leopoldo; \\ Meschengieser, Gonzalo Germán
}

\section{Sergio Leandro Agoff}

sergioleandroagoff@gmail.com

Universidad Nacional de General Sarmiento,

Argentina

\section{Leopoldo Blugerman}

sergioleandroagoff@gmail.com

Universidad Nacional de General Sarmiento,

Argentina

Gonzalo Germán Meschengieser

gonzalo_meschengieser@aysa.com.ar

Agua y Saneamientos Argentinos, Argentina

\author{
Documentos y Aportes en Administración Pública y \\ Gestión Estatal \\ Universidad Nacional del Litoral, Argentina \\ ISSN: $1666-4124$ \\ ISSN-e: 1851-3727 \\ Periodicidad: Semestral \\ vol. 21, núm. 36, 2021 \\ Editor: José Vigil | jvigil@fce.unl.edu.ar \\ Recepción: 14 Junio 2021 \\ Aprobación: 02 Agosto 2021
}

URL: https://doi.org/10.14409/daapge.2021.36.e0014

\section{(1) (2)(2)}

Esta obra está bajo una Licencia Creative Commons AtribuciónNoComercial-CompartirIgual 4.0 Internacional.

Para citar este artículo: Para citar este artículo:Agoff, S. L.; Blugerman, L.; Meschengieser, G. G. (2021) "La gestión pública de empresas: el desafío de un abordaje multinivel” DAAPGE Vol. 21, $N^{\circ}$ 36, 2021, pp. 124-145. UNL, Santa Fe, Argentina.
Resumen: El artículo desarrolla la idea de que la gestión pública de empresas se da en distintos niveles, incorporando una consideración sobre la cuestión territorial, entendida como una "escala de base", no siempre debidamente reflejado en los estudios. El trabajo supone que el desafío de la gestión pública de las empresas es articular tres niveles: en primer lugar, el global, atendiendo a las cuestiones de la experiencia internacional, los modelos de desarrollo y la cuestión de la inserción e integración regional; en segundo lugar, el territorial, enfocando la vinculación con actores públicos y privados en los espacios específicos de desarrollo de su actividad; y, por último el organizacional, abordando la cuestión de la combinación entre estructura y profesionalización que permita un trabajo sostenible en los otros dos niveles. El artículo apela a fuentes secundarias, principalmente y discute a partir de la referencia provista por la literatura especializada.

Palabras clave: gestión pública de empresas 1, niveles de gestión , paradigma socio-territorial.

Abstract: The article expands the idea that the public management of companies occurs at different levels, integrating a consideration of territorial issues, understood as a "base scale", not always accurately reflected in previous studies on the matter. The paper assumes that the challenge of the public management of companies is to articulate three levels: first, the global, taking into account the questions of international experiences, development models and the question of regional projection and integration; secondly, the territorial, focusing on the link with public and private actors in the specific areas of their activity; and, finally, the organizational, addressing the question of blending between structure and professionalization that might allow sustainable work at the other two levels. The article mainly appeals to secondary sources and discusses from the references provided by the specialized literature.

Keywords: State-owned enterprises management, Management levels, Socio-territorial paradigm. 


\title{
1. Introducción ${ }^{1}$
}

La discusión sobre modelos de desarrollo y el papel que le cabe al Estado en esos procesos ha subtendido el debate político y económico desde el nacimiento mismo del capitalismo, aceleradamente desde el surgimiento de la revolución industrial (Polanyi y Sánchez, 1975). En ese marco, todas las teorías de la política y la economía -las corrientes clásicas y neoclásicas, el keynesianismo, el liberalismo político, el marxismo, etc.- han tomado posición y desarrollado, incluso en sus variantes internas, enfoques sobre la cuestión.

Esos debates alcanzaron en los países centrales a partir del último tramo del siglo XIX a las nacientes organizaciones público-estatales encargadas de la provisión de servicios e infraestructura para el desarrollo capitalista. Puede decirse que, de allí en adelante y en un movimiento del centro a la periferia, así se inició la discusión sobre el rol de esas organizaciones públicas -que cobraron la forma empresarial- en cuanto a su tamaño, magnitud, capacidad y calidad de intervención.

Dicho esto, la discusión contemporánea sobre la cuestión adquiere nuevo impulso a partir de las crisis económicas en la década de 1970 del siglo pasado, en la que, en paralelo a un cambio en el patrón productivo, comienza un ciclo de revisión profunda de la estructura estatal y, con ella, de la gestión pública de empresas.

Como afirma Mabel Thwaites Rey, "durante los años de auge del neoliberalismo, el papel del estado empresario fue objeto de un cuestionamiento radical, y la privatización de empresas públicas se convirtió en la receta ineludible para las endeudadas periferias capitalistas" (Thwaites Rey; 2017, 42). Luego de esa experiencia, extendida en las últimas dos décadas del siglo pasado, desde los comienzos del Siglo XXI en América Latina se recupera el protagonismo estatal y la gestión pública de empresas vuelve a ser considerada en los esquemas de desarrollo que los gobiernos de corte nacional-popular o progresista intentaron llevar adelante, con diferencias y similitudes.

Por su parte Guillermo Guajardo Soto, sostiene que

\begin{abstract}
"la historia de la intervención estatal en América Latina, a pesar de haber sido clave en el desarrollo de la región, hoy en día presenta lagunas notables sobre el papel y trayectoria de las empresas públicas en el siglo XX, tema que debe ser retomado por varias razones. La primera es por la re-emergencia de las empresas estatales en el sector energético internacional, especialmente en petróleo, gas y electricidad. La segunda es la evidencia empírica que indica que en la región latinoamericana las empresas públicas siguen cumpliendo un papel relevante en sectores estratégicos, al igual que en Asia y Europa. Finalmente, una tercera razón es que tras décadas de privatizaciones actualmente hay una débil memoria colectiva sobre estos organismos, predominando la versión neoliberal que justificó la venta del acervo público". (Guajardo. 2013; p7)
\end{abstract}

También "del otro lado del Atlántico" ese debate se expresa en una contestación a las posiciones neoliberales. Como lo refiere Thwaites Rey (2017), es la economista italiana Mariana Mazzucato en su libro El Estado Emprendedor (2014), quien apunta críticamente a la teoría neoclásica que sostiene que el papel del estado debe limitarse a proveer la infraestructura básica, educación, salud y seguridad e intervenir en la economía solo "por default", argumentando que 
"en la práctica, sin embargo, el estado innova no sólo garantizando sino también creando los mercados, mientras que los capitalistas privados se limitan a sacar beneficios de las iniciativas del estado. (...) La verdadera fuente de la creación de riqueza, muestra la investigadora italiana, son los estados, que financian las investigaciones de base sobre las que se encaraman luego los empresarios. Es decir, es la acumulación colectiva y el financiamiento público lo que explica la creación de riqueza en el capitalismo." (Thwaites Rey, 2017, 45)

Este último período, no obstante, no se ha cursado sin reveses o contrariedades. De hecho, como lo señala la propia Thwaites Rey (2017), los cambios en las correlaciones de fuerzas políticas han dado lugar al desarrollo en la región de gobiernos promercado que intentan desandar aquel camino.

Este trabajo se propone desplegar un abordaje de la gestión empresarial pública a partir de la identificación de niveles de la gestión, en la que distinguimos a) el nivel global, que resume los problemas a nivel internacional, tanto general como continental, que asume la necesidad de una puesta en sintonía con diversas corrientes y experiencias, y la posibilidad de consolidación de políticas generales de mediano plazo; b)el nivel territorial, es decir, el de la articulación de las empresas como actor social en relación con sus poblaciones de referencia, tanto público general, como proveedores y también organizaciones sociales de base territorial. Este nivel representa una novedad en los enfoques, porque se propone abordar un conjunto de relaciones no siempre consideradas en los análisis; y c)el nivel organizacional, el que remite no sólo al orden de las combinaciones de estructura y tecnología para la realización de los propósitos de las empresas en la producción de bienes y servicios, sino a la configuración sociotécnica necesaria para que esa producción pueda alinearse también con los objetivos y definiciones de los otros dos niveles, esto es, que suministre una capacidad de producción, al tiempo que una capacidad de lectura de los compromisos socioterritoriales de la acción organizacional.

Finalmente, a modo de conclusiones provisorias, se enunciará una propuesta de articulación de los tres niveles mencionados, a modo de un modelo o paradigma de gestión pública de empresas.

\section{El nivel global}

\subsection{Los estadios de la gestión pública de empresas: de la caída de la URSS al Covid19}

Como consecuencia de una sucesión de crisis económicas que impactaron en gran parte del planeta, la década del 70 del siglo pasado dio origen a un proceso de transformación profundo de la visión del rol del Estado empresario. No obstante, el hecho histórico de la caída de la Unión Soviética a comienzos de la década de 1990, tal como lo señalara Eric Hobsbawm (2005), constituyó un momento de quiebre político y marcó, respecto de lo que nos ocupa, el comienzo de una nueva etapa para las empresas públicas en el mundo. Hasta ese momento dos polos parecían organizar la actividad económica a nivel planetario. Por un lado, los países en los que los Estados intervenían de manera regulada en la economía, dejando a otros actores del mercado el rol de asignar los recursos y dinamizar la economía; y, por el otro, aquellos en los que prevalecía la máxima participación estatal en la economía, concentrando los medios de producción 
y comercialización. Las empresas públicas, hasta ese fin del Siglo XX corto en palabras del historiador, tenían dos tipos de desarrollo diferenciado: ocupando sectores estratégicos en el primer grupo, o componiendo todo el entramado productivo y de servicios en el segundo. Se trataba de un escenario bipolar, en el que el análisis se encontraba, de algún modo, "encapsulado", tal como lo muestran las discusiones suscitadas por el llamado proceso de "desestatalización" en la URSS. Ese debate constituyó una discusión profunda, en cuánto a cuál sería el mejor camino para consolidar la política de la Perestroika y hasta qué punto se podrían rearticular algunos de los pilares del régimen anterior ${ }^{2}$ con el que se hallaba en ciernes en los países que integraban el Pacto de Varsovia.

La desintegración de la Unión Soviética dio inicio al reacomodamiento del sector empresario público a nivel global. Casi de forma simultánea a la integración al mundo capitalista de las organizaciones productoras de bienes y servicios en el Este se producían transformaciones profundas en el Oeste. Las sucesivas crisis financieras que acaecieron en el período, y particularmente la de las subprimes en 2007, reintrodujo la cuestión a partir de la gran intervención de los Estados en la economía, la mayor desde el Plan Marshall, especialmente en los EE. UU. (Szalavetz, A.,2015; Heyes, J., Lewis, P., \& Clark, I., 2012; Franke, M. M., 2014).

A partir de entonces puede decirse que se produce una reorganización en el espectro de las empresas con participación estatal. Se hace más heterogéneo el planteo del papel estatal y de su intervención en el mundo empresario, diversificándose las formas de vínculo entre capital y Estado. Los Estados retoman la intervención, mediante la conformación de empresas o las participaciones accionarias, en nuevos bienes y servicios, antes exclusivos del sector privado. Se trata, entre otros sectores, de las nuevas tecnologías de la información, tanto en su versión software como hardware. Internet comienza a postularse como bien público, como ya se había prefigurado anteriormente (Hallgren, M. M., \& McAdams, A. K., 1997; Stiglitz, J. E., 1999) y los insumos para la fabricación de dispositivos tecnológicos, como el litio, la plata o el coltán, recursos estratégicos de los países con las mayores fuentes. La globalización, que tornó difusas las fronteras económicas y culturales entre países, anteriormente fundamento de los corrimientos de las fronteras en favor de una menor participación pública, opera ahora en un sentido inverso, es decir, como una corriente de articulación internacional que vincula corporaciones y Estados, acelerando la reasignación de capitales en los mercados financieros, promoviendo la logística de productos para el comercio internacional y produciendo nuevos tipos de migraciones acompañando la dislocación de clúster productivos y la consolidación de sistemas de outsourcing, configurando una nueva arquitectura económica de gobernanza global (Messner (2004). En ese contexto las empresas de gestión pública también rediseñaron su ámbito de acción, impulsando muchas de ellas verdaderos procesos de internacionalización que continúan hasta el día de hoy (Landoni, M., 2018; Xie, E., \& Tang, Q., 2018; Mariotti, S., \& Marzano, R. 2020).

La Pandemia de Covid-19 podría significar un nuevo estadio del proceso iniciado en la última década del Siglo XX. Se trata de una catástrofe a nivel global que cataliza la transformación digital de los procesos de relacionamiento y generación de valor a nivel mundial y deja expuesta la frágil situación del planeta en términos humanitarios, de gobernanza global y ecológicos. Las empresas de gestión pública, incluyendo aerolíneas, empresas de tecnologías, productoras de 
vacunas y bancos de desarrollo, inician un nuevo ciclo. Esta nueva etapa incluye también, como "novedad", estrictos mecanismos de rendición de cuentas para mostrar el mejor uso de los recursos. ${ }^{3}$ Szarzec, Dombi, y Matuszak (2021), para el caso de Europa, muestran una fuerte correlación entre marco institucional, crecimiento económico y gestión pública de empresas llegando a la conclusión de que el impacto de las empresas públicas en el crecimiento económico está condicionado a la calidad de las instituciones (gubernamentales) de manera positiva: mejores instituciones implican un efecto de crecimiento más favorable de las empresas estatales.

Después del rápido repaso a un período rico e intenso en transformaciones, conviene, también sintéticamente, establecer la importancia económica del sector de empresas de gestión pública en el mundo.

En un artículo publicado en el portal VOXEU, Büge, Egeland, Kowalski y Sztajerowska $(2013)^{4}$ aportan algunos datos acerca de las magnitudes y tendencias involucradas en los estudios existentes sobre este sector empresarial. Para empezar, establecen que más del 10\% de las empresas más grandes del mundo son de propiedad estatal (204 empresas), proviniendo de 37 países diferentes. Sus ventas conjuntas ascendían a 3,6 billones de dólares en 2011. Esto representa más del $10 \%$ de las ventas combinadas de la totalidad de Forbes Global 2000 y equivale al 6\% del PIB mundial, superando los PIB de países como Alemania, Francia o Reino Unido

Asimismo, un informe de julio de 2018 del IFC del Banco Mundial ${ }^{5}$ establece que Las empresas de propiedad estatal (EPE) representan el 20 por ciento de la inversión, el 5 por ciento del empleo y hasta el 40 por ciento de la producción nacional en países de todo el mundo. Ofrecen servicios críticos en sectores económicos clave, incluidos los servicios públicos, las finanzas y los recursos naturales.

\subsection{Estado, empresas de gestión pública y desarrollo}

Las opciones históricas de la teoría económica han tematizado la cuestión tomando a la empresa pública como objeto discreto, dando base conceptual a los fenómenos históricos reseñados anteriormente.

Según Rozas Balbontin y Bonifaz (2014), la teoría económica ha discutido sobre el origen y funcionamiento de las empresas públicas, en el marco de desafíos de desarrollo de los países. Para lo que denominan enfoque tradicional, la creación de empresas públicas "obedecería a factores que se relacionan principalmente con la estructura del mercado y la dinámica de su funcionamiento, y abarcaría sólo tangencialmente aspectos vinculados al papel del Estado en la construcción económica y social de los países" (Rozas Balbontín y Bonifaz, 2014; 30). La perspectiva tradicional validó también la creación de empresas públicas en condiciones de "monopolio natural". En todo caso, ese tipo de enfoques neoclásicos insisten, en cuanto a la legitimidad de la intervención estatal en las llamadas "fallas de mercado".

Por su parte Chang (2007), reconoce que el mercado es un mecanismo poderoso en la promoción del desarrollo económico, pero en el que deben reconocerse a menudo "fallas" que necesitan corrección. La provisión de bienes 
públicos, como tarea principal, no puede limitarse a una correcta política impositiva y de distribución. Es necesaria, además, una regulación adecuada, que enmarque a los mercados para impedir que las lógicas cortoplacistas de búsqueda de beneficios afecten la inversión de largo plazo imprescindible para un desarrollo económico sustentable. ${ }^{6}$ Sin encontrar "evidencias teoréticas" contundentes a favor o en contra de las empresas de gestión pública, Chang sostiene que ellas pueden cumplir un papel decisivo a la hora de la inversión de largo plazo, si superan los problemas de "management" que muchas veces las aquejan.

En los debates posteriores, discutiendo a las corrientes neoclásicas, la legitimad del papel del Estado como controlante o, directamente, propietario de empresas y activos productivos no se vincularía al funcionamiento del mercado, como en las teorías desarrollistas. Como afirman Rozas Balbontín y Bonifaz, entre los argumentos que legitiman la intervención estatal en el ámbito empresarial, están los que se ligan a los objetivos de interés público o razones de utilidad pública "y que se relacionan con la necesidad de completar los mercados de bienes y servicios mediante el desarrollo de algunas actividades que no son suficientemente atractivas para los agentes privados o, en su defecto, que estos no pueden desarrollar por motivos de índole económica, financiera o tecnológica" (Rozas Balbontín y Bonifaz, 2014; 30).

Así, comenzó a concebirse a la empresa pública como un instrumento de política de desarrollo, correctora de desequilibrios estructurales, afirmando su papel como mecanismo de reasignación de recursos y redistributivo. Ese papel corrector y equilibrador procura resolver insuficiencias en la estructura productiva originadas en la insuficiencia del capital disponible (o asignado) y en la precariedad del desarrollo de conocimiento y de tecnologías De ese modo, la gestión estatal persiguió completar cadenas productivas o bien buscó la promoción tecnológica en sectores industriales; o bien se orientó a la provisión de servicios de infraestructura económica, considerados esenciales para el desarrollo de las demás actividades productivas.

Entre las décadas del 50 y 70 del siglo pasado se afirmó una corriente de "teoría de las empresas públicas”, basada en dos principios centrales: la posibilidad de la coexistencia de empresas públicas y privadas en el mismo espacio económico; y el rol que las empresas públicas tienen en el desarrollo de las políticas públicas. Entre los antecedentes (o "afluentes") de esta teoría, Rozas Balbontín y Bonifaz destacan las posiciones de Oskar Lange y Abba Lerner, que en las décadas de 1930 y 1940 habían desarrollado un enfoque de lo que se denominó "socialismo de mercado". Esta perspectiva postulaba

"que se podian preservar las ventajas funcionales de la organización empresaria capitalista (en especial, aquellas referidas a la eficiencia productiva y a la descentralización de las estructuras de producción), evitando las distorsiones derivadas del poder de mercado y ciertas consecuencias sobre la distribución del ingreso que se consideraban indeseables" (Rozas Balbontín y Bonifaz, 2014; 32)

Los avances ulteriores de la teoría de la empresa, según los autores mencionados, retomaron dos aspectos principales: el de las distorsiones en el sector privado y el de las consideraciones distributivas, entendiendo a la empresa pública como factor de equilibrio en ambos asuntos. 


\subsection{La gestión pública de empresas en el mundo}

En esta nueva etapa de la historia se plantea la necesidad imperante del establecimiento de prioridades de los Estados para intervenir en la economía. Desde un punto de vista analítico, la experiencia internacional propone entonces que la cuestión de la marcha de la gestión pública de empresas a nivel global sea abordada siguiendo la ruta de algunos interrogantes, como los que se despliegan debajo:

- ¿En la producción de qué bienes públicos y globales deberían intervenir los estados?

- ¿Podrían existir verdaderas empresas públicas de origen multipolar capaces de asignar recursos de forma equitativa a los países en peores condiciones de hacerlo por sus medios (experiencia del Fondo de Acceso Global para Vacunas Covid-19, COVAX)?

- ¿Cuál es el límite para la participación de capitales estatales en la producción de bienes y servicios en empresas de gestión pública del extranjero sin inmiscuirse en conflictos ligados a la soberanía?

- ¿Cuál será la consideración final que tendrán los nuevos productos y servicios vinculados a las tecnologías de la información (5G, redes sociales, etc.) y, por ende, necesitados de participación estatal?

- ¿Cómo iniciar el camino de la transformación de la explotación de recursos naturales, a través de la gestión pública de empresas, a modelos de desarrollo inclusivo y sostenible?

- ¿Cómo establecer un sistema que destaque a aquellas empresas a nivel global que han intervenido con éxito en mercados potencialmente dañinos para el desarrollo humano (por ejemplo, tabaco o alcohol en Suecia)?

Contemplando la casi segura diversidad de respuestas que un debate de esos términos puede dar, sí es seguro que a nivel global la gestión pública de empresas considerará las siguientes cuestiones:

- Nuevos sectores de intervención: a los sectores clásicos de intervención del Estado a través de empresas, fundamentalmente los de servicios públicos como suministro de energía y agua potable; infraestructura vial y transporte; y defensa, se incorporan otros vinculados a las tecnologías de la información y financieros. Tendido de redes de fibra óptica, puesta en órbita de nuevos servicios satelitales, extracción y comercialización de materias primas para la producción de dispositivos tecnológicos y desarrollo de Fintech son algunos de los mercados en los que pueden comenzar a operar las empresas de gestión pública.

- Internacionalización de las empresas de gestión pública: con renovada vocación de operar en latitudes lejanas, impulsadas por las variables financieras como los tipos de cambio y la conformación de nuevos mercados por la transformación sociodemográfica y económica de los países emergentes, las empresas estatales se lanzan a conquistar nuevos espacios de intervención. Patrimonio de las petroleras y compañías telefónicas, sectores como el sanitario, impulsado por nuevas ramas 
como la nanotecnología o las industrias del entretenimiento de capitales estatales pueden comenzar a operar como verdaderas multinacionales. También son factores para considerar los intercambios entre países bajo una especie de "diplomacia" de empresas de gestión pública o empresas privadas avaladas por los estados mediante los cuales pueden intercambiarse productos manufacturados por primarios, lo que incluso genera interrogantes sobre cuestiones vinculadas a la soberanía.

- López Morales y Nava Aguirre (2018), trabajando sobre el caso de PEMEX, establecen un marco general para pensar los procesos de internacionalización, mostrando sus distintas variantes, y comparando las modalidades para empresas privadas y empresas de gestión pública. En cuanto a esto, señalan que los procesos son muy similares, caracterizándose por su gradualidad y secuencialidad.

- Capitales de origen mixto: con las nuevas formas jurídicas en las conformaciones de las sociedades en muchos países del mundo, existen cada vez más empresas con capitales mixtos, con mayoría estatal o empresaria. Además, la necesidad de contar con nuevas fuentes de financiamiento que acompañe los procesos de expansión e internacionalización que mencionamos, lleva a muchas de estas compañías a cotizar en diversos mercados financieros internacionales. Por otra parte, nuevos tipos de instrumentos financieros podrían ser implementados en un marco de evolución catastrófica del cambio climático, intentando llevar previsibilidad a los precios de los productos primarios. Un ejemplo claro es el de los futuros ligados al agua y varios minerales.

- Profesionalización de sus RRHH y dispositivos tecnológicos: la pandemia de Covid-19 es el catalizador de un proceso corporativo que ya estaba en marcha: la implementación de sistemas de teletrabajo y la digitalización de las operaciones. Éstas traen aparejadas varias consecuencias, entre las que se destacan la posibilidad de expansión por fuera de las fronteras de los países y probablemente aumentos en la productividad y la eficiencia. También se incrementarán los riesgos de ciberataques y la dependencia a la infraestructura digital. El personal de las empresas debe acompañar estas transformaciones con nuevos conocimientos y herramientas de gestión, más aún si se cumple con lo expuesto más arriba respecto de la intervención en la competencia de mercados novedosos.

- Lógica de producción dislocada y transnacional: la apertura de las economías y los cambios en las políticas monetarias en muchos países del mundo han generado la tendencia a dislocar la producción de productos y servicios buscando la eficiencia en términos de logística y competitividad en función de los tipos de cambio (Messner 2004). Un trabajo colectivo de la Fundación $1^{\circ}$ de Mayo en Europa (Leonardi Salvo et al 2012), advierte sobre las consecuencias en el campo de las relaciones laborales de los Acuerdos Transnacionales de Empresas, realizados en el marco de la Unión Europea.

Estos mecanismos de producción transnacional pueden también alcanzar las operaciones de las empresas de gestión pública en diversos sectores de la economía; el citado trabajo coloca en el foco del desarrollo de esos mecanismos, 
la articulación con otros actores económicos sectoriales y las organizaciones sindicales correspondientes.

A manera de síntesis de este primer nivel proponemos este cuadro que trabaja sobre tres dimensiones: el sector de actividad de la gestión pública de empresas, el tipo de articulación de la acción estatal y los resultados dados (o previstos, como en el "post-Covid-19"),

\begin{tabular}{|c|c|c|c|}
\hline & SECTOR & ARTICULACIÓN & RESULTADOS \\
\hline $\begin{array}{l}\text { Post caída } \\
\text { de la URSS. } \\
\text { Auge } \\
\text { neoliberal }\end{array}$ & $\begin{array}{l}\text { Apertura } \\
\text { fuerte hacia } \\
\text { el sector de } \\
\text { servicios y } \\
\text { financiero }\end{array}$ & $\begin{array}{l}\text { El Estado "se } \\
\text { retira" en las } \\
\text { economias. } \\
\text { Fuertes } \\
\text { transformaciones } \\
\text { en la ex URSS y } \\
\text { reconfiguración } \\
\text { en China. Se dan } \\
\text { procesos de } \\
\text { privatización de } \\
\text { empresas en } \\
\text { distintos países }\end{array}$ & $\begin{array}{l}\text { El proceso de } \\
\text { globalización } \\
\text { integra servicios }\end{array}$ \\
\hline $\begin{array}{l}\text { Posterior a } \\
\text { la crisis de } \\
\text { las } \\
\text { "Subprimes" }\end{array}$ & $\begin{array}{l}\text { Ampliación } \\
\text { de los } \\
\text { sectores en } \\
\text { los cuales se } \\
\text { opera }\end{array}$ & $\begin{array}{l}\text { Los Estados } \\
\text { intervienen } \\
\text { remedialmente } \\
\text { en las economias } \\
\text { de mercado }\end{array}$ & $\begin{array}{l}\text { Empresas de } \\
\text { gestión pública } \\
\text { adoptan la } \\
\text { cultura de } \\
\text { multinacionales. } \\
\text { Se incorpora } \\
\text { con fuerza la } \\
\text { participación en } \\
\text { la } \\
\text { comercialización } \\
\text { de los } \\
\text { productos y } \\
\text { servicios. }\end{array}$ \\
\hline $\begin{array}{l}\text { Post Covid- } \\
19\end{array}$ & $\begin{array}{l}\text { Incorporación } \\
\text { de las } \\
\text { empresas } \\
\text { públicas en } \\
\text { productos, } \\
\text { servicios y } \\
\text { desarrollos } \\
\text { vinculados a } \\
\text { las nuevas } \\
\text { tecnologias y } \\
\text { estilos de } \\
\text { vida }\end{array}$ & $\begin{array}{l}\text { Se fortalece la } \\
\text { intervención } \\
\text { estatal }\end{array}$ & $\begin{array}{l}\text { Cadenas de } \\
\text { producción } \\
\text { transnacionales. }\end{array}$ \\
\hline
\end{tabular}

\section{El nivel territorial}

Como señalábamos previamente, todas estas transformaciones se dan en un marco en el que, en el último cuarto del siglo pasado, a la luz de un cambio en el patrón de acumulación productiva, desde el fordismo a un esquema esbelto-posfordista/toyotista (Boltanski y Chiappello 2002, Coriat 1994), se alumbró un proceso de creciente interdependencia y aumento en los flujos económicos, informativos y culturales; en breve, la globalización (Keohane y Nye 1998, Nye 2002). Paralelamente con dicho proceso de mutación, se advierte el menguado rol del Estado nacional como actor central en la arena social y económica (Salamon 1994). Esto aconteció en un marco en el que las fronteras entre organizaciones pasan a ser cada vez menos claras, pasándose de una lógica 
mecánica y estandarizada, que internalizaba todas sus actividades, a una lógica de articulación por proyectos, en red (Recio 2000), adhocrática (Mintzberg 2005).

Es a inicios de este nuevo milenio en que la praxis y la conceptualización sobre el rol del Estado en la arena social se reconfigura (por ejemplo, Mazzucatto 2014), pero en cualquier caso la lógica reticular en la vinculación del Estado con otros actores, en particular de la sociedad civil sigue siendo un elemento central del paisaje, que, no obstante, ha sido categorizado y problematizado de diversas formas.

Cuando la colaboración se refiere a la labor conjunta entre organizaciones de la sociedad civil y actores gubernamentales, el término utilizado con frecuencia es Partnership / Asociación, la que se puede definir como una

\begin{abstract}
Relación entre diversos actores, basada en objetivos mutuamente acordados, perseguida a través de un entendimiento compartido de la división del trabajo más racional basada en las respectivas ventajas comparativas de cada socio. Esta relación da como resultado una influencia mutua, con un cuidadoso equilibrio entre la sinergia y la autonomía respectiva, que incorpora el respeto mutuo, la participación equitativa en la toma de decisiones, la responsabilidad mutua y la transparencia (Brinkerhoff 2002b: 14, traducción propia).
\end{abstract}

Más estrictamente, al Partnership también se lo define como un acuerdo "entre una organización del sector público y cualquier organización fuera del sector público" (Bovaird 2004: 200), por lo que el término no es exclusivo de las articulaciones del ámbito de la sociedad civil, sino que el eje es la articulación del ámbito público, con actores privados o del Tercer Sector. Dos características fundamentales han sido reconocidas en los partnerships/ asociaciones: tienen identidad organizacional -lo que se quería crear con la articulación y que aún no existe en cada una de las organizaciones que participarán- y mutualidad un sentido de igualdad percibida entre los socios, a pesar de sus diferencias (Brinkerhoff 2002: 1135)-.

Cuando la literatura especializada analiza la colaboración en este sentido, hay dos dimensiones comunes en todas las definiciones relevadas: el primero es el porqué de las colaboraciones entre las organizaciones, y la respuesta tiende a ser teleológica. Las colaboraciones son procesos que tienden a estructurar un mecanismo que sirva para alcanzar mejor una serie de objetivos que cada organización no puede cumplir por sí sola: por ejemplo, para lograr sus propios objetivos o comunes a ambos (Mattesich et al 2001), o para "Permitir que las entidades asociadas alcancen resultados que excedan los que podrian lograrse sobre la base de los recursos individuales de cada participante". (Yankey et al.2001, traducción propia), entre otras.

En segundo lugar, la literatura indica lo que se necesita para mantener la relación colaborativa, que a su vez se descompone en dos factores: uno de los factores, estructurales - para generar mecanismos y condiciones para sostener la colaboración-: por ejemplo, con objetivos mutuamente acordados, perseguidos a través de una comprensión compartida de la división más racional del trabajo basada en las ventajas comparativas de cada socio (Brinkerhoff 2002a y 2002b), y un segundo factor, intangible, la generación de una cultura común entre los asociados a través del desarrollo de una "identidad colectiva compartida" (Fox 2010), una "identidad organizacional" (Brinkerhoff 2002b), etc. 
Como se sugería al inicio del acápite, la clave organizacional se desplazó del control y la centralización en una gran empresa-organización a un mayor esfuerzo (reticular) de coordinación entre asociados multisectoriales cada vez más delgados. La articulación entre los actores de los diversos sectores sociales parece ser la norma para hacer frente a la complejidad del entorno contemporáneo. Así, en la actualidad nos encontramos ante un panorama organizacional que podríamos denominar híbrido en la dinámica asociativa voluntaria, y entonces:

Las lineas que delimitan el sector han sido objeto de desafios y revisiones con frecuencia, ya que los fondos disponibles y las responsabilidades se han desplazado de un lado a otro entre las organizaciones empresariales, sin fines de lucro y gubernamentales. Alcanzar un consenso sobre la definición misma de sector voluntario y sin fines de lucro es difícil porque muchas de las caracteristicas y actividades centrales de las organizaciones sin fines de lucro se superponen y compiten cada vez más con las de las empresas y el gobierno. (Frumkin 2002: 1 -traducción propia).

Los límites difusos entre los diversos sectores sociales (Brandsen 2010: 839), son una característica cada vez más presente en la dinámica de las OSC, y son a la vez tanto la causa como la consecuencia de los esfuerzos de colaboración entre éstas y los actores públicos.

\subsection{Desafíos de la gestión en la colaboración entre Estado y actores sociales:}

Este proceso empezó a observarse de manera creciente, como señalamos arriba, con el retiro del Estado Nacional de esferas sociales a finales del siglo pasado, al momento en que realizó una especie de tercerización, adelgazamiento (Boltanski y Chiapello 2002) o delegación de dichas actividades, en un marco de reforma estatal, privatización y achicamiento del Leviatán (así, Salamon 1994, o la miríada de literatura sobre New Public Management-Hood 1991, por ejemplo-, entre muchos otros, dan cuenta de este fenómeno). Bienvenidos al Estado esbelto trabajando vinculado con las OSC (amplio paraguas que incluye diversas formas organizacionales en su interior).

En primer lugar, Young (2000: 149) hace hincapié en que esta relación entre OSC y el Estado puede entenderse de una de las siguientes maneras: a) que operan de forma independiente, como suplementos, b) que trabajan como complementos entre sí, o c) se vinculan con el gobierno en una relación de confrontación, ejerciendo accountability mutua.

Por otro lado, Coston (1998) analiza el fenómeno de manera más general, y desarrolla una tipología de la relación entre OSC y -ya no Estado sino- gobierno, a través de estas tres dimensiones: a) el grado de aceptación del pluralismo institucional que tenga el gobierno; b) el equilibrio de poder en esta relación; y finalmente c) el grado de formalidad y el nivel de vinculación con el gobierno. Esto da como resultado un continuo en el vínculo, que va desde, inicialmente, la represión a la rivalidad, de allí a la competencia, a la contratación, el thirdparty government, luego a la cooperación, la complementariedad y como punto de llegada, la colaboración.

Bryson (et al 2006) llega a la conclusión de que las colaboraciones intersectoriales cuentan con más probabilidades de éxito cuando son capaces de crear uno o más mecanismos de vinculación, como promotores del acuerdo, que sean igualmente poderosos para ambas partes, un acuerdo general sobre 
cuál es el problema que debería articular conjuntamente a OSC y gobierno, o que haya redes existentes en marcha en el momento de la formación inicial del "partenariato".

Aun cuando sea posible diferenciar en un nivel analítico el set actor-agencia, la naturaleza del contexto político importa y mucho cuando se trata de analizar las relaciones entre el Leviatán y las OSC. El trabajo entre los actores políticos y las OSC creció considerablemente en las últimas cuatro décadas, como resultado de los procesos de retiro o redefinición del Estado de bienestar (Salamon 1994), como ya repetimos.

Adicionalmente, por lo general, con mayor o menor nivel de explicitación, la literatura sobre colaboración, escrita desde el norte global, considera desde un punto de vista no sólo liberal, sino también hegeliano, que la sociedad civil es el locus de las OSC, y que dicho loci está en algún lugar "entre" el Estado (lo público) y el mercado (lo privado), y que ninguno de los dos actores "ocupa" todo el espacio social. Cuando no hay actores públicos (o privados) que ocupen todo el espacio social, existe la posibilidad de la emergencia de actores que representen los diversos intereses comunitarios de la sociedad civil, esto es, las OSC. Por consiguiente, no extraña que la mayor parte del análisis de las relaciones entre el Estado y las OSC partan del presupuesto de que hay margen para que el Estado no ocupe todo el espacio público, por lo que se han centrado en el estudio de dicha relación en un marco democrático (Coston, Bryson, Brinkerhoff no han problematizado la variable "régimen político" por caso). Cabe interrogarse, en ese sentido, qué miradas sobre el fenómeno se pueden traer desde nuestro sur global para problematizar el fenómeno con categorías más ancladas en nuestra dinámica sociohistórica.

También aquí a modo de síntesis y anticipación, ubicamos el desarrollo de las dimensiones de articulación y resultados:

\begin{tabular}{|c|c|c|}
\hline & ARTICULACIÓN & RESULTADOS \\
\hline Posfordismo & $\begin{array}{l}\text { Creciente } \\
\text { interdependencia } \\
\text { y aumento en } \\
\text { los flujos } \\
\text { económicos, } \\
\text { informativos y } \\
\text { culturales }\end{array}$ & $\begin{array}{l}\text { Tercerización, } \\
\text { adelgazamiento } \\
\text { o delegación } \\
\text { de dichas } \\
\text { actividades, en } \\
\text { un marco de } \\
\text { reforma } \\
\text { estatal, } \\
\text { privatización. } \\
\text { Estado esbelto }\end{array}$ \\
\hline $\begin{array}{l}\text { Reconfiguración } \\
\text { de comienzos } \\
\text { del Siglo XXI }\end{array}$ & $\begin{array}{l}\text { Partnership } \\
\text { mecanismos de } \\
\text { vinculación, } \\
\text { acuerdo general } \\
\text { sobre cuál es el } \\
\text { problema que } \\
\text { debería articular } \\
\text { conjuntamente } \\
\text { a osc y } \\
\text { gobierno }\end{array}$ & $\begin{array}{l}\text { Hibridez. } \\
\text { Relaciones } \\
\text { entre OSC y el } \\
\text { Estado: a) } \\
\text { como } \\
\text { suplementos, } \\
\text { b) } \\
\text { complementos } \\
\text { entre si, o c) } \\
\text { accountability } \\
\text { mutua. }\end{array}$ \\
\hline
\end{tabular}




\section{El nivel organizacional}

Tanto las preguntas sobre los efectos organizacionales de la multipolaridad de capitales, como la concepción reticular, que se reseñaban en los niveles global y socioterritorial, respectivamente, demandan una traducción en el campo de las organizaciones concretas.

Recientemente, se ha predicado sobre la gestión pública de las empresas, en torno a su capacidad de respuesta en situaciones críticas, algunas propuestas sostenidas en corrientes organizacionales que apuntan a la idea de "gobierno corporativo de las empresas", basadas en la experiencia inglesa del informe Cadbury en 1992. ${ }^{7}$ En ese sentido, McDonald afirmó que "la corporatización es como un lobo con piel de cordero, que ofrece una fachada de propiedad pública mientras propaga la ideología de mercado y la acumulación privada, y todo ello sin tener que asumir los riesgos económicos y políticos asociados a las formas tradicionales de privatización." (Chavez, 2013.; p289)

El desarrollo de esa capacidad se juega en terrenos muy concretos, esto es, qué competencias tienen las empresas para decidir en torno a su modelo de gestión y construir a partir de ello sus estructuras. Si puede resultar cierto que esa mirada subraya una perspectiva instrumental del abordaje sobre empresas de gestión pública, no es menos cierto que las decisiones practicadas en ese terreno operan consecuencias muy concretas también.

\subsection{La capacidad productiva de la organización y los problemas de estructura organizacional}

Como lo afirma Guajardo (2013), históricamente la empresa pública desplegó una diversidad de formatos y denominaciones como compañías, empresas, institutos, sociedades, etc. de carácter centralizado o descentralizado, dependiente de la administración pública de nivel nacional, provincial o municipal. La diversidad de formatos organizacionales y jurídicos forman parte del núcleo de la discusión sobre el papel de las empresas estatales, y Guajardo destaca su complejidad y la interpretación divergente sobre ello y, más en general, el rol de los Estados en los modelos de desarrollo.

Una de las discusiones que atravesaron el campo del debate sobre el rol estatal y del mercado en los procesos de desarrollo fue el que estableció una dicotomía entre la gestión por resultados y la gestión por procesos. Las posiciones reconocidas en la Nueva Gerencia Pública fueron las que más insistieron en la oposición de una gestión que se orientase "al ciudadano", incorporando el instrumental de la management proveniente de distintas experiencias de grandes empresas y corporaciones del sector privado. La crítica tradicional a la gestión estatal subrayaba el carácter "procedimentalista" del hacer burocrático en el ámbito público, al tiempo que promovía su remoción por una lógica que persiguiera de manera directa los resultados finales de las políticas, eliminando lo que se entendía como obstáculos en ese sentido.

Jorge Hintze (2003) retoma de manera específica la cuestión, desplegando un conjunto de consideraciones que muestran su complejidad y el modo en que esa crítica, tan asociada a los procesos de reforma estatal acaecidos en nuestra 
región, y particularmente en nuestro país, la simplificaba indebidamente. Desde su perspectiva, no hay tal dicotomía, o, mejor dicho, si cabe analíticamente la distinción de los dos términos, no es lícito oponerlos como si fueran horizontes valorativos enfrentados. Sin buenos procesos, no hay buenos resultados, sería su conclusión. Para llegar a esa afirmación Hintze despliega una serie de distinciones conceptuales que son muy útiles para pensar este nivel organizacional de la gestión pública de empresas.

Por un lado, la distinción entre tipos de producto. Es decir, cuando se habla de "resultados", se hace referencia a un tipo particular que es el "producto externo", es decir, aquella producción que una organización "externaliza", hace circular en el mercado, sea bien o servicio. Pero para que esa producción tenga lugar, es indispensable que exista un tipo de resultado intermedio, que denomina "producto interno", esto es, los que funcionan como insumo para la producción final de la organización. Todo el movimiento de las áreas de apoyo de una organización a sus unidades productivas es la que genera esos productos internos, como condición de la producción externa. Pero para esas áreas de apoyo, su encadenamiento con las áreas sustantivas implica un sistema de relaciones, de procesos que entrañan también un tipo de producción, la de los llamados "productos organizacionales", destinados al mantenimiento, modificación o ampliación de esas relaciones. De modo que esta primera distinción coloca a los procesos en el seno mismo de los resultados. En segundo lugar, el trabajo de Hintze distingue distintas modalidades de gestión ordenadas a partir de cómo se asignan los recursos, a qué están dirigidos. Se identifican tres modalidades de gestión organizacional: operativa; "por proyectos"; y "por programas".

Las combinaciones planteadas por Hintze entre modalidades de gestión y estructura, parecen muy pertinentes para construir una mirada, a la vez que son instrumentos para el diseño, para distintas alternativas de empresas públicas. En ello, parece decisiva la evaluación de los sectores de intervención estatal y el tipo de producción involucrada, todo esto orientado por la definición del modelo de desarrollo al que se aspira. Constituye un caso interesante que ilustra bien este punto el de INVAP, que es una empresa constituida, en su mayoría, por especialistas en distintas áreas dedicados a la fabricación de dispositivos tecnológicos. Su modalidad de trabajo es por proyectos, es decir, que fabrican productos individuales o unitarios y en plazos establecidos. Como lo establecen Seijo y Cantero (2012), INVAP en su estructura combina las áreas verticales tecnológicas con áreas horizontales transversales de servicio, haciendo uso del repertorio del conocimiento tecnológico y organizacional existente

\subsection{La burocracia en (de) las empresas de gestión pública}

La "cuestión burocrática" en las empresas de gestión pública retoma los tópicos que habitualmente se ordenan tanto en la recuperación positiva del concepto, como en su uso en una línea crítica.

Para Schweinheim (2011), la burocracia aparece como una herramienta de escala en un plano de discusión política acerca de modelos socio-económicos y vuelta a enfocar, con nueva luz, la cuestión del papel de los Estados y sus distintos niveles de gobierno. Schweinheim propone pensar la burocracia, como criterio organizativo en el contexto de la discusión política y se centra en el 
reposicionamiento del Estado como un actor central para una estrategia de desarrollo nacional. Parte de la preocupación por resituar al Estado como un jugador potente en la estrategia de desarrollo económico y social. Esta vuelta al "centro del ring" de este peleador que había sido desplazado durante los años 80 y 90, fundamentalmente en esa última década cuando se consolidó la presencia del llamado "Consenso de Washington" (Williamson 2009).

El resultado organizacional de la implantación acrítica de formas institucionalistas, en las décadas del 80 y 90, fue la forma "adhocrática" que refiere Mintzberg (1993), forma privilegiada frente a contextos "inestables". Hubo un "deslumbramiento" con esta forma organizacional, una "moda" al decir del mismo autor, solidaria a su vez con la preeminencia de las ideas del New Public Managment (gestión por resultados, gestión por proyectos, etc.).

\section{3 ¿Dónde ubicar en este planteo a las empresas de gestión pública?}

Con estos materiales, podría pensarse una estructura organizacional que combinar al mismo tiempo la manda política de la provisión de bienes, la inclusividad y universalidad, con la flexibilidad para la resolución de problemas de la producción de esos bienes, determinados por cambios contextuales (del mercado, políticos, tecnológicos, sociales) que no pueden atenderse de manera jerárquica, atado a un corpus normativo cerrado y un conjunto de procedimientos rígidos. Es decir, una combinación, en los términos de Mintzberg, de burocracia y adhocracia. Pero ihay una forma organizacional que pueda contemplar semejante combinación? ¿Qué tipo de burocracia se conformaría en ese caso?

Para una posible respuesta a esas preguntas, retomamos el enfoque propuesto por Luisina Perelmiter (2016): nos referimos en particular al concepto de "burocracia plebeya".

Perelmiter repara en la cuestión de la distancia social (distancia institucional) que supone la estructura y el comportamiento burocráticos, mirando cómo, desde cierto marco de políticas, esa distancia puede trastocarse. Su trabajo propone un abordaje que puede ser remitido a lo que en Mintzberg aparece bajo la idea de coaliciones internas y externas. Se trata del "descubrimiento" de la variedad de grupos y actores que conforman la "planta", cada uno con sus lógicas e intereses. Ese primer trabajo que hace el texto es central, porque permite ver lo que constituye una parte fundamental: la idea de no homogeneidad o, para decirlo por la positiva, de la alta heterogeneidad de toda organización. En este caso, al tratarse de una organización pública estatal de primerísimo nivel, la heterogeneidad se vuelve un valor político de altísimo grado de criticidad. Perelmiter lo nombra como "mosaico ministerial". 8

Nuestro argumento central es, pues, que la burocracia plebeya subvierte jerarquias y recrea la promesa de proximidad, pero al mismo tiempo produce un "efecto distancia", como cualquier otra burocracia. Es precisamente es a contradicción la que define su lógica de funcionamiento. (Perelmiter; 2016; 18-19-20)

Esa "lógica de funcionamiento", contradictoria, vuelve a colocar la cuestión del manejo de la incertidumbre, de la membrecía, y de la resolución de conflictos, como elementos de la dinámica organizacional. 
El siguiente cuadro formula una síntesis, considerando las dimensiones de articulación y resultados.

\begin{tabular}{|l|l|l|}
\hline & ARTICULACION & RESULTADOS \\
\hline $\begin{array}{l}\text { Estructura } \\
\text { organizacional }\end{array}$ & $\begin{array}{l}\text { Integración } \\
\text { divisional y por } \\
\text { proyectos }\end{array}$ & $\begin{array}{l}\text { Capacidades } \\
\text { de producción } \\
\text { de bienes } \\
\text { públicos }\end{array}$ \\
\hline \multirow{3}{*}{ Burocracias } & $\begin{array}{l}\text { Racionalidad } \\
\text { medios-fines. } \\
\text { Relación entre } \\
\text { actores internos } \\
\text { y externos en } \\
\text { coaliciones }\end{array}$ & $\begin{array}{l}\text { Provisión de } \\
\text { bienes } \\
\text { públicos a } \\
\text { escala. } \\
\text { Reducción de } \\
\text { las distancias } \\
\text { institucionales }\end{array}$ \\
\hline
\end{tabular}

\section{Conclusiones: el desafío de la construcción de un nuevo modelo de gestión pública de empresas}

Si no puede afirmarse un nuevo cambio en el patrón de acumulación capitalista, idéntico al producido en el último cuarto del Siglo XX, tampoco puede negarse que ciertas reconfiguraciones han tenido lugar con el advenimiento del Siglo XXI.

Tal como señalan Regalsky y Rougier (2015) hay temas o cuestiones que han reaparecido, retomando algunas del pasado: el papel del Estado en el desarrollo; los criterios para medir su desempeño y las características específicas de la gestión empresarial pública. Los embates sufridos en el período del auge neoliberal y las ideas y prácticas a las que dio lugar comenzaron a ser discutidas con la crisis de los gobiernos que llevaron adelante esas políticas, primero en América Latina y luego, a partir de la crisis de las hipotecas en 2008 en EE. UU. y Europa.

Pero esa discusión no desconoció las críticas que las perspectivas neoclásicas colocaron en el escenario del debate. La reaparición de la gestión pública de empresas debió consignar la experiencia traumática en la dimensión de la articulación público-privado que sucesivamente vio dificultades para desarrollarse, sea por la lógica rentística de los privados o las propias resistencias burocráticas estatales (Regalsky y Rougier, 2015). Simultáneamente, debió dar cuenta de los problemas de eficiencia, transparencia y competitividad y calidad en la administración, tal como lo desarrollan Schlarek Curuchet, Re Crespo y Torres (2011), argumentando a favor del reposicionamiento estatal en los procesos de desarrollo económico de los países.

Una de las cuestiones que, en el marco de esta reaparición de temas, vuelve a concitar la atención refiere al conjunto de problemas que Horacio Boneo desarrolló en distintos (y ya clásicos) trabajos de las últimas décadas del siglo pasado sobre la decisión en la empresa pública. Esos trabajos parten de lo que se entiende como una definición relacional de la empresa pública, en la medida que destaca dos tipos de vinculación principal de la misma: las transacciones económicas, fruto de su intervención en un campo de actividad específico; y las relaciones de poder, que para Boneo son las que emergen de la particular ligazón 
entre gobiernos y empresas. De modo que la gestión empresarial pública debe ser colocada en el marco del análisis de la trama de relaciones en la que se inserta.

Haciendo foco en el mismo asunto, Jeanniot y Bourgault (1992), trabajando sobre el caso de Air Canadá, desarrollaron un modelo marco para la toma de decisiones. En él establecían que el proceso de decisión, en una empresa de este tipo, es producto del encuentro y confrontación de cuatro dimensiones: 1) la misión y objetivos empresarios; 2) el estatuto y estrategia organizacional; 3) la relación con la estructura económica y el mercado; 4) la relación con el contexto (social, político y cultural). El peso relativo de esas dimensiones, su combinación, perfila un cauce específico de decisión.

Las decisiones tomadas a partir de la consideración de esas cuatro dimensiones se clasifican en estratégicas, tácticas y operativas, considerando por un lado el impacto en el tiempo del contenido de la decisión; y por el otro, el nivel de responsabilidad que toma la decisión. Si las decisiones tácticas y operativas se resumen en la articulación de los elementos internos de la organización, las decisiones de carácter estratégico son el resultado del análisis de situaciones externas e internas. Son decisiones de largo plazo y constituyen el marco de las otras. Nacen de la interrogación sobre la relación con los entornos y sobre la satisfacción que las empresas obtienen de esas relaciones. Para Jeanniot y Bourgault ese tipo de decisiones dan lugar a objetivos de oportunidad, poniendo en juego las fortalezas de las empresas y objetivos de desafío, evidenciando la necesidad de cambios o reforzamientos de sus capacidades. Este enfoque, que se despliega en el marco del modelo de empresa estatal clásica, es un prolegómeno interesante para el planteo de la gestión pública de empresas.

Las observaciones detalladas en los acápites de este trabajo dan cuenta de elementos críticos que pueden organizarse en una matriz, vinculando las cuestiones de los tres niveles: global, territorial y organizacional. La definición de sectores estratégicos, la articulación de capitales de origen mixto, las estrategias de internacionalización, la constitución de dispositivos híbridos y de partenariado, las alternativas de diseño organizacional por proyectos y la integración interna de los "cuerpos" profesionales y burocráticos, son algunos de esos elementos que podrían configurar un modelo de gestión pública de empresas.

Las tres dimensiones consideradas en las síntesis de los puntos anteriores constituyen ejes a partir de los cuales organizar esos elementos en la construcción del modelo. En primer lugar, el sector, atendiendo a los campos de actividad específica en los que se combinan demandas sociales, recursos estratégicos y tecnológicos; en segundo lugar, la modalidad de articulación, atendiendo a la variedad de actores, sus intereses y lógicas de vinculación; y, por último, en tercer término, los resultados de gestión, que expresan la producción alcanzada, su distribución y los impactos en términos de desarrollo.

En el modelo de gestión pública de empresas que se sugiere, cada una de las dimensiones se trabaja en los tres niveles detallados, dotando de complejidad a la acción organizacional.

En el siguiente cuadro se procura presentar aspectos centrales de esa complejidad. Los elementos que se detallan en la operación sobre el sector en los niveles territorial y organizacional, que no aparecían en los cuadros de síntesis anteriores, son derivados de las definiciones estratégicas alcanzadas en el nivel global. 


\begin{tabular}{|c|l|l|l|}
\hline $\begin{array}{l}\text { Dimensión / Nivel de } \\
\text { Gestión }\end{array}$ & SECTOR & ARTICULACIÓN & RESULTADOS \\
\hline GLOBAL & $\begin{array}{l}\text { Definición } \\
\text { política de } \\
\text { sectores } \\
\text { estratégicos }\end{array}$ & $\begin{array}{l}\text { Integración de } \\
\text { capitales. } \\
\text { Definición de } \\
\text { socios } \\
\text { estratégicos }\end{array}$ & $\begin{array}{l}\text { Desarrollo } \\
\text { económico }\end{array}$ \\
\hline TERRITORIAL & $\begin{array}{l}\text { Establecimiento } \\
\text { de formas } \\
\text { institucionales } \\
\text { de actores entegración } \\
\text { el territorio } \\
\text { de dispositivos } \\
\text { públicos } \\
\text { híbridos }\end{array}$ & $\begin{array}{l}\text { Resolución } \\
\text { de problemas } \\
\text { de bienes y } \\
\text { servicios }\end{array}$ \\
\hline ORGANIZACIONAL
\end{tabular}

De este modo, tres elementos constituyen el núcleo de la propuesta de abordaje de la gestión pública de empresas multinivel: las tendencias a una producción multilocalizada, que supone la integración económica internacional en unidades mayores; la articulación asociativa pluriactoral en el territorio, que integra la heterogeneidad de actores sociales relevantes y de sus intereses; y las dos direcciones de la estructura organizacional, esto es, la escala y la racionalidad en la relación medios-fines, que asegura estándares de calidad y eficiencia productiva, por un lado; y la proximidad, es decir, la capacidad de adecuarse a situaciones contextuales y "entrar en diálogo" con actores sociales de referencia de la acción empresarial, por el otro.

El escenario ha mutado: empresas públicas nacionales que se articulan en cadenas de valor globales; organizaciones de la sociedad civil que, ante cambios en la forma de representación, pasaron a tener un gran peso en la representación de amplios sectores populares. Así, la gestión pública de empresas entonces se presenta no sólo como el emergente de una dinámica de mayor alcance, como fruto de cambios productivos, sociopolíticos y organizacionales, sino que es, asimismo, una fuerza que vuelve a reconfigurar dicha dinámica.

Volvemos a preguntar: ¿̇el escenario ha mutado realmente? La pregnancia de la imagen de las políticas estatales como insertas en una estructura de arenas (Oszlak y O 'Donnell, 1976) parece seguir siendo un potente descriptor del fenómeno. Las burocracias plebeyas, pero atentas al juego global, las organizaciones sociales locales articulándose con actores nacionales e internacionales, públicos y privados, y con un gran peso en la representación de sectores, a nuestro juicio, sólo parecen complejizar el escenario tensivo planteado por Oszlak y O 'Donnell en los albores del último golpe de Estado sufrido por nuestro país.

En definitiva, en este regreso del Estado, el nuevo paradigma en ciernes busca llamar la atención sobre la mayor complejidad de las tensiones ínsitas en la arena política actual, y llevar adelante sus actividades en múltiples niveles de gestión, desde el micro al macro, desde el territorio a la cadena de valor global, a través de un equilibrio entre eficacia y eficiencia, entre burocracia maquinal y adhocracia. Busca balancear el cumplimiento de las demandas sociales con la sostenibilidad, 
pivoteando entre la planificación estratégica y la emergente, pragmático, pero sin perder de eje su rol como proveedor central de bienes públicos.

\section{Agradecimientos}

Este articulo responde a la convocatoria realizada por la Red Universitaria de Carreras de Administración y Política Pública (RUCAPP). Editores invitados: Sergio De Piero (Universidad Nacional de la Plata y Universidad Nacional Arturo Jauretche) y Elsa Beatriz Pereyra (Universidad Nacional de General Sarmiento y Universidad Nacional de San Martín).

\section{Referencias}

Berger, G., L. Blugerman, R. Petrov C. Guo \& D.H. Smith (2016) "Relationships and collaboration among associations", in Smith, D.H. (Ed.) Palgrave Handbook of Volunteering, Civic Participation and Nonprofit Associations, London: Palgrave. Pp. 1162-1185.

Boltanski, L. y E. Chiappelo (2002) El nuevo espiritu del capitalismo. Madrid: Ediciones Akal.

Brandsen, T. (2010) "Hybridity/Hybridization" en International Encyclopedia of Civil Society, Anheier, H. K., S. Toepler, R. List, Editors. New York, NY: Springer Science + Business Media.

Brinkerhoff, J. M. \& D. W. Brinkerhoff (2002) "Government-nonprofit relations in comparative perspective: evolution, themes and new directions". Public Administration \& Development; Feb. 22, 1; 3-18.

Brinkerhoff, J. M. (2002) “Government-nonprofit partnership: a defining framework". Public Administration \& Development. Feb. 22, 1; 19-30.

Bryson, J. M., B. C. Crosby, M. M. Stone (2006), “The Design and Implementation of Cross-Sector Collaborations: Propositions from the Literature". Public Administration Review, December (Special Issue): 44-55.

Chang H. J. (2007) State-owned enterprise reform. New York, June 2007 Copyright $(\odot$ United Nations DESA

Chavez D. (2013) "Empresas Públicas: transformaciones, desafíos y oportunidades: seminario internacional, Montevideo, octubre de 2012", en Revista de Gestión Pública ISSN: 0719-1820 Vol. II Número 1; Enero-Junio 2013

Coriat, B. (1994) El taller y el cronómetro. Madrid: Siglo Veintiuno Editores.

Coston, J. M. (1998) "A model and typology of government-NGO relationships". Nonprofit and Voluntary Sector Quarterly, 27(3), 358-382.

Fox, J. (2010) “Coalitions and Networks", en International Encyclopedia of Civil Society, Anheier, H. K., S. Toepler, R. List, Editors. New York, NY: Springer Science + Business Media.

Franke, M. M. (2014). "State intervention in times of the global economic crisis", en: Public Administration and the Modern State (pp. 75-89). London: Palgrave Macmillan.

Frumkin, P. (2002). On being nonprofit: A conceptual and policy primer. Cambridge, MA: Harvard Univ. Press. 
Guajardo Soto G. (2013) "Empresas Públicas en América Latina: historia, conceptos, casos y perspectivas", en Revista de Gestión Pública ISSN: 0719-1820 Vol II Número 1; Enero-Junio 2013

Hallgren, M. M., \& McAdams, A. K. (1997). "The economic efficiency of Internet public goods", en Internet economics, 455-478

Heyes, J., Lewis, P., \& Clark, I. (2012). "Varieties of capitalism, neoliberalism and the economic crisis of 2008?”, en: Industrial Relations Journal, 43(3), 222-241.

Hintze J. (2003) "Gestión por procesos y resultados en el aparato estatal: una cuestión abierta". Ponencia presentada en el SEGUNDO CONGRESO ARGENTINO DE ADMINISTRACIÓN PÚBLICA - "Sociedad, Gobierno y Administración Pública. Reconstruyendo la estatalidad: Transición, instituciones y gobernabilidad ", AAEAP. Córdoba, República Argentina, Noviembre.

Hobsbawm, E. (2005) Historia del siglo XX 1914-1991. Barcelona: Crítica.

Hood, Christopher (1991) “A public management for all seasons?", Public Administration 69:1 (3-19).

Keohane, R. O. and J. S. Nye (1998) "Power and interdependence in the information age". Foreign Affairs. 77 (5): 81-94.

Jeanniot P. y J. Bourgault (1992), "Air Canadá y la decisión en la empresa pública” en Guiot, J. y A. Beaufils Diseño de la Organización. Del cargo a la megaestructura. Legis. Bogotá Colombia.

Landoni, M. (2018). "Corporatization and internationalization of state-owned enterprises: The role of institutional intermediaries", en International Journal of Public Sector Management: Redding, K. S.

Leonardi Salvo et al (2012), Acuerdos Transnacionales de Empresas. Doc N 58. Fundación $1^{\circ}$ de Mayo, Centro Sindical de Estudios. Madrid. España

López- Morales J.S y Nava- Aguirre K.M (2018) The road to internationalization of a latin state company: the case of Pemex GCG: revista de globalización, competitividad y gobernabilidad, ISSN 1988-7116, Vol. 12, No. 2, 2018, págs. 97-114.

Mariotti, S., \& Marzano, R. (2020). "Relational ownership, institutional context, and internationalization of state - owned enterprises: When and how are multinational co - owners a plus?”, en Global Strategy Journal, 10(4), 779-812

Mattessich, P. W.; M. Murray-Close and B. R. Monsey (2001) Collaboration: What Makes It Work, $2^{\circ}$ ed. St. Paul, MN: Amherst H. Wilder Foundation.

Mazzucato, M. (2014). El Estado emprendedor. España: RBA LIBROS.

Messner, D. (2004). "Regions in the "world economic triangle", en Local Enterprises in the Global Economy-Issues of Governance and Upgrading. Cheltenham.Edward Elgar Publishing Limited, 20-52.

Mintzberg, H. (2005) La estructuración de las organizaciones. Barcelona: Ariel.

Nye Jr, J. S. (2002) The Paradox of American Power. Why the World's Only Superpower Can't Go It Alone. New York: Oxford University Press.

Oszlak, O., \& O'Donnell, G. (1976). Estado y politicas estatales en América Latina: hacia una estrategia de investigación. Buenos Aires: Centro de Estudios de Estado y Sociedad (CEDES). Documento G.E. CLACSO/ No4. Extraído de: https://repositorio.cedes.org/bitstream/123456789/3332/1/ Doc_t04.pdf (fecha de acceso: 28/07/2021).

Perelmiter, L. (2016) Burocracia plebeya. La trastienda de la asistencia social en el Estado Argentino. UNSAM EDITA. San Martín, Provincia de Buenos Aires 
Polanyi, K., \& Sánchez, A. (1975). La gran transformación. Juan Pablos.

Recio A. (2000). "Empresa red, distribución de la renta y relaciones laborales", ponencia presentada en las VII Jornadas de Economía Crítica de la Universidad Castilla la Mancha. Albacete 3-5 febrero. Extraído de: http://pendientedemigracion.ucm.es /info/ec/jec7/pdf/com2-9.pdf (fecha de acceso: 06/05/2016).

Regalsky A. y M. Rougier (2015) Introducción en Los derroteros del Estado empresario en la Argentina. Siglo XX. Eduntref. Buenos Aires

Rozas Balbontín, P. y J. L. Bonifaz F. (2014) Notas sobre la teoría de la empresa pública de servicios de infraestructura y su regulación Santiago de Chile: CEPAL Publicación de las Naciones Unidas ISSN 1680-9017 LC/L.3793

Salamon, L. M. (1994) “The Rise of the Nonprofit Sector", Foreign Affairs. 73 (4):109-122.

Schclarek, A., S. Re Crespo y L. A. Torres (2011), Errores del pasado. Soluciones a futuro. CIPPES, Buenos Aires

Schweinheim, G. (2011) "Desarrollo, instituciones y organización pública. Niveles de gobierno, reglas institucionales y tipologías organizacionales para el desarrollo", en J. A. Bonifacio (comp.) Reformas administrativas y politicas públicas. Enfoques, instrumentos y prácticas en Argentina. AAEAP. 2013. Buenos Aires

Seijo, G. L y J. H. Cantero (2012) ¿Cómo hacer un satélite espacial a partir de un reactor nuclear? Elogio de las tecnologías de investigación en INVAP. Revista REDES Vol $18 \mathrm{~N}^{\circ} 35$ Instituto de Estudios sobre la Ciencia y la Tecnología UNQUI. http:// www.unq.edu.ar/advf/documentos/526a8c38ec2b5.pdf

Stiglitz, J. E. (1999). “Knowledge as a global public good", en: Global public goods: International cooperation in the 21st century, 308, 308-325.

Szalavetz, A. (2015). "Post-crisis approaches to state intervention: New developmentalism or industrial policy as usual?", en Competition \& Change, 19(1), 70-83.

Szarzec, K. Dombi, Á. \& Matuszak, P., (2021). "State-owned enterprises and economic growth: Evidence from the post-Lehman period," Economic Modelling, Elsevier, vol. 99(C).

Thwaites Rey, M. (2017) "Encrucijadas del Estado y las Empresas Públicas en una América Latina en disputa", en Estado abierto Revista sobre el Estado, la administración y las políticas públicas, Vol. 1 No 3, abril-julio 2017 - Buenos Aires: INAP - ISSN 2525-1805

Williamson, J. (2009). A Short History of the Washington Consensus. Law and Business Review of the Americas, 15(1), 7-23.

Xie, E., \& Tang, Q. (2018). "Institutionalization to internationalization: The transformational dynamics and outward foreign direct investment of state-owned enterprises", en International Journal of Public Sector Management.

Yankey, J. A., B.W. Jacobus \& K.M. Koney (2001) Merging Nonprofit Organizations: The Art and Science of the Deal, Cleveland, OH: Mandel Center for Nonprofit Organization.

\section{Notas}

1 Este articulo responde a la convocatoria realizada por la Red Universitaria de Carreras de Administración y Política Pública (RUCAPP). Editores invitados: Sergio De Piero (Universidad Nacional de la Plata y Universidad Nacional Arturo Jauretche) y Elsa 
Beatriz Pereyra (Universidad Nacional de General Sarmiento y Universidad Nacional de San Martín).

2 https://elpais.com/diario/1991/06/25/internacional/677800813_850215.html (fecha de acceso: 30/07/2021).

3 https://www.imf.org/pt/News/Articles/2020/05/06/blog-state-owned-enterprises-i n-the-time-of-covid-19 (fecha de acceso: 30/07/2021).

4 State-owned enterprises in the global economy: Reason for concern? Max Büge, Matias Egeland, Przemyslaw Kowalski, Monika Sztajerowska 02 May 2013 VOX Eu CEPR

5 State-Owned Enterprises IFC, julio de 2018

6 "For example, 20 times more money is spent on research on slimming drugs than on research on malaria, a disease that kills more than a million people every year. If we want a broad-based and politically sustainable development, we need to find mechanisms that can meet the basic needs of everyone" (Chang, 2007; 6).

7 El Informe Cadbury se publicó en 1992 como respuesta a los escándalos corporativos de BCCI, Polly Peck y Maxwell, y fue seguido por la creación del Código de Gobierno Corporativo del Reino Unido.

8 El trabajo de Perelmiter aborda el caso del Ministerio de Desarrollo Social de la Nación entre 2006 y 2008. 